\title{
Metabolomics study on the therapeutic effect of the Chinese herb pair Fructus Aurantii Immaturus and Rhizoma Atractylodis Macrocephalae in constipated rats based on UPLC-Q/TOF-MS analysis
}

\author{
Shuai Yan ${ }^{1,2 \#}$, Min Hao ${ }^{3 \#}$, Huiju Yang ${ }^{4 \#}$, Mingming Sun ${ }^{2}$, Bensheng Wu ${ }^{2}$, Yinzi Yue ${ }^{2}$, Xiaopeng Wang ${ }^{2}$ \\ ${ }_{1}^{1}$ Jiangsu Key Laboratory for Pharmacology and Safety Evaluation of Chinese Materia Medica, School of Pharmacy, Nanjing University of Chinese \\ Medicine, Nanjing, China; ${ }^{2}$ Suzhou TCM Hospital Affiliated to Nanjing University of Chinese Medicine, Suzhou, China; ${ }^{3}$ School of Pharmacy, \\ Zhejiang Chinese Medicine University, Hangzhou, China; ${ }^{4}$ Department of Anorectal Surgery, The Third Affiliated Hospital of Henan University of \\ Traditional Chinese Medicine, Zhengzhou, China \\ Contributions: (I) Conception and design: S Yan; (II) Administrative support: S Yan, M Hao, H Yang; (III) Provision of study materials or patients: Y \\ Yue, X Wang; (IV) Collection and assembly of data: M Sun; (V) Data analysis and interpretation: B Wu; (VI) Manuscript writing: All authors; (VII) \\ Final approval of manuscript: All authors. \\ \#These authors contributed equally to this work. \\ Correspondence to: Yinzi Yue; Xiaopeng Wang. Department of Anorectal Surgery, Suzhou TCM Hospital Affiliated to Nanjing University of Chinese \\ Medicine, Suzhou 215009, China. Email: yueyinzi@126.com; wxpeng2004@163.com.
}

Background: In China, Zhishi (Aurantii Fructus Immaturus) - Baizhu (Atractylodis Macrocephalae Rhizoma) is a well-known herb pair used to treat gastrointestinal motility disorders for thousands of years, and it has especially shown a definite advantage in the treatment of slow transit constipation (STC). However, the mechanism of Zhishi-Baizhu (ZSBZ) in the treatment of STC remains unclear. In this study, plasma metabolomics research combined with metabolic pathway analysis has been used to illuminate the potential mechanism of its effects against STC.

Methods: Parameters of intestinal transit ratio, plasma motilin (MTL), substance P (SP), adenosine triphosphate (ATP), histological alteration of the colon and MLCK expression in the colon were detected to evaluate the effects with respect to STC. Principal component analysis (PCA) was used to investigate the global metabolite alterations, while orthogonal partial least squares discriminant analysis (OPLS-DA) and t-test were used to filter potential metabolite markers. Moreover, metabolic pathway analysis was employed.

Results: Oral administration of ZSBZ significantly prevented the development of STC.

It increased the expression of MTL and SP in serum, as well as the expression of ATP and MLCK in the colon. ZSBZ administration alleviated symptoms in loperamide-induced constipated rats, evidenced by the increase of intestinal transit ratio. Futhermore, 9 potential biomarkers of STC were screened, and the levels were all reversed to different degrees after ZSBZ administration. Metabolic pathway analysis showed that the improvement of STC by ZSBZ was mainly related to caffeine and vitamin B6 metabolism.

Conclusions: Our study identifies the metabolic networks of constipated rats and demonstrates the efficacy of this metabolomics approach to systematically study the therapeutic effects of ZSBZ on constipation.

Keywords: Aurantii Fructus Immaturus; Atractylodis Macrocephalae Rbizoma; herb pair; slow transit constipation (STC)

Submitted Jan 23, 2020. Accepted for publication Jul 13, 2020.

doi: $10.21037 /$ apm-20-280

View this article at: http://dx.doi.org/10.21037/apm-20-280

^ ORCID: 0000-0003-1053-8018. 


\section{Introduction}

Constipation is a widespread functional gastrointestinal disorder among humans in modern society and seriously influences people's health and quality of life $(1,2)$. According to the current pathophysiology mechanism, constipation caused by functional diseases can be divided into various types, such as defecatory disorder, mixed constipation, normal transit constipation and slow transit constipation (STC) (3). Among them, STC is the most usual type of chronic constipation and is characterized by slow proximal colonic transit and obviously increased total bowel transit time (4). According to previous study, several reasons may cause STC, including structures, machinery, metabolism, or functional factors (5). Despite its high morbidity, the precise pathogenesis of STC remains unknown $(6,7)$.

In China, Zhishi (Aurantii Fructus Immaturus) and Baizhu (Atractylodis Macrocephalae Rbizoma) are a traditional herb pair utilized to regulate qi, harmonize the stomach, promote digestion, resolve lumps and release distension which was first described by the renowned Chinese physician Zhang Zhongjing (150-219 A.D. in the Eastern Han Dynasty) in Essentials from the Golden Cabinet (Fin Gui Yao Lue), a medical classic. This herb pair combination therapy was widely used to treat gastrointestinal motility disorders for thousands of years, especially showing a definite advantage in the treatment of STC. Previous clinical studies have shown that the Zhishi-Baizhu (ZSBZ) herb pair can effectively enhance gastrointestinal motility, increase the frequency of bowel movements, shorten the transit time of the colon, reduce the symptoms and physical signs of constipation patients, improve the anorectal dynamics index, and improve the living quality of patients (8). However, because its clinical effect is the result of various components targeting multiple metabolic pathways, the mechanism of ZSBZ in the treatment of STC remains unclear.

Metabolomics, a branch of systems biology, is a holistic analytical method for characterizing small molecular endogenous metabolites in various biological samples (9). In recent years, metabolomics has been widely used in TCM research, including the compatibility mechanism exploration of active constituents in Chinese medicine $(\mathrm{CM})$, processing mechanism of $\mathrm{CM}$ and toxicity mechanism of $\mathrm{CM}$, because its research mode is similar to the mechanism of TCM and it provides a new paradigm to discover the complicated mechanisms in TCM (10). Ultra-performance liquid chromatography equipped with electrospray ionization quadrupole time-of-flight mass spectrometry detection
(UPLC-Q/TOF-MS) combined with multivariate statistical analysis is the most common method in metabolomics research due to the extensive availability of the technology and compatibility with the extensive separation of biological samples (11-13). In this study, plasma metabolomics combined with metabolic pathway analysis has been used to elucidate the therapeutic mechanism of ZSBZ in STC from a metabolic perspective.

We present the following article in accordance with the ARRIVE reporting checklist (available at http://dx.doi. org/10.21037/apm-20-280).

\section{Methods}

\section{Chemicals and reagents}

The HPLC grade methanol, MS grade acetonitrile and formic acid were purchased from Merck (Darmstadt, Germany). Pure water was obtained from Jilin Wahaha Food Co. Ltd. (Jilin, China). Ultrapure water was prepared from a Milli-Q water purification system (Millipore, Bedford, MA, USA). Loperamide Capsules were from Xian Janssen Pharmaceutical Ltd. Mosapride Citrate Capsules were from Jiangsu Hengrui Pharmaceutical Co., Ltd. Chloral hydrate was obtained from China National Pharmaceutical Group Co., Ltd. Rabbit monoclonal antibody against MLCK was supplied by Sigma (USA). Acacia gum was purchased from Guangzhou Nanfang Chemical Glass Co., Ltd. Activated carbon was from Hangzhou Wood General Factory. The super centrifuge was from Eppendorf (Germany). The microplate reader was from Thermo Fisher Scientific (USA). An Olympus light microscope was employed (BX41, Japan). The slicer was the RM 2235 from Leica Instruments (Germany). The electric constant temperature air blower dryer was from Shanghai Botai Experimental Equipment Co., Ltd.

\section{Sample preparation}

Zhishi (Lot: 170910) was purchased from Suzhou Tianling Traditional Chinese Medicine Decoction Pieces Co., Ltd. (Suzhou, China). Baizhu (Lot: 17C821) was obtained from Suzhou Boyuan Pharmaceutical Co., Ltd. (Suzhou, China). The aqueous extract of ZSBZ was prepared as follows: $2 \mathrm{~kg}$ of Zhishi and $1 \mathrm{~kg}$ Baizhu were mixed with 10 times the amount of distilled water and soaked for $0.5 \mathrm{~h}$. Second, the mixture was boiled with mild heat for $40 \mathrm{~min}$ and afterwards filtered through 4 layers of carbasus. This 
method was repeated to extract the residue with 6 times the amount of distilled water. Afterwards, two aqueous extracts were merged and vacuum-concentrated to $1 \mathrm{~g} / \mathrm{mL}$ of crude drug by a rotary evaporator. The samples were stored at $4{ }^{\circ} \mathrm{C}$ for future use.

\section{Animal study design and induction of constipation}

The study was approved by ethics board of Suzhou TCM Hospital Affiliated to Nanjing University of Chinese Medicine (NO. 2018-03-216), in compliance with national and institutional guidelines for the care and use of animals. Forty healthy, specific pathogen-free (SPF), 5-week-old, male Sprague-Dawley rats (170-180 g) were purchased from the Jiangsu Laboratory Animal Centre in Suzhou, China [license approval number: SCXK (Su) 2017-0007]. All rats were maintained in an animal house (SPF degree) with a barrier system assisted with a clean laminar flow chamber in the Experimental Animal Center of Nanjing University of Chinese Medicine and used for experimentation after 1 week. Feeding environment parameters were as follows: room temperature, $26^{\circ} \mathrm{C}$; relative humidity, 45-55\%; light condition, 12 h light/dark cycles; noise level, <60 dB; clean and quiet, well-ventilated. All experimental operations in this study were carried out in accordance with the Provision and General Recommendation of the Chinese Laboratory Association. The protocol was approved by the Medical Research Committee on Animal Care and Use, Nanjing University of Chinese Medicine. Animals were randomly divided into four groups: control group, Lop group, mosapride group and ZSBZ group (ZSBZ water extract). Except for the control group, the other three groups were induced as the STC models by oral administration of $3 \mathrm{mg} / \mathrm{kg}$ loperamide suspension twice per day (09:00 and 17:00) for 14 days, while the control group rats were administered normal saline only (14). ZSBZ group rats were intragastric administered ZSBZ water extract $(81 \mathrm{mg} / \mathrm{kg})$ twice a day for 14 days. Mosapride group rats were intragastric administered with $1.58 \mathrm{mg} / \mathrm{kg}$ mosapride suspension twice per day for 14 days. On day 14, all rats were intraperitoneally injected with $3 \%$ chloral hydrate $0.5 \mathrm{~h}$ after the last portion. Blood samples were collected by the abdominal aortic method.

\section{Analysis of body weight, feeding conditions and fecal excretion}

The body weight, food intake, and water consumption of rats in each group was measured daily during the experimental period. We collected fecal pellets from individual rats $24 \mathrm{~h}$ after the treatment administration. The total number, wet weight, and water content of the fecal pellets were detected. All measurements were performed in triplicate to ensure accuracy.

\section{Measurement of intestinal transit ratio}

On day 14, all rats were fasted for $12 \mathrm{~h}$ and allowed free access to water, then fed charcoal in $10 \%$ acacia gum. After $0.5 \mathrm{~h}$, the rats' abdomens were opened and the intestines were removed from the pylorus to the ileocecal junction. The total length of the truncated intestine and the charcoal transport distance were measured, and then the intestinal motility rate was calculated by the following formula: charcoal transit ratio $(\%)=$ distance traversed by the charcoal $(\mathrm{cm}) /$ total length of small intestine $(\mathrm{cm}) \times 100 \%$.

\section{Histological observation}

The experimental rats were euthanized and sacrificed, and the transverse colons were removed and cut into 8- to $10-\mathrm{mm}$ sections. The transverse colons were gently shaken in PBS to remove the contents of the intestine, then fixed with $10 \%$ neutral-buffered formalin for more than $24 \mathrm{~h}$. After fixation, the tissues were embedded in paraffin, and embedded tissues were cut into 5 - $\mu$ m thick sections. All of the sections were then stained with hematoxylin-eosin (H\&E). The colon tissue was then embedded, sectioned, and HE stained. The thickness of the transverse colonic mucosa and the morphology of the cells in the colon were observed under binocular light microscopy (Carl Zeiss, Germany).

\section{Measurement of neurotransmitter serum concentration}

First, $5-10 \mathrm{~mL}$ of blood from the abdominal aorta was injected into the EDTA anticoagulant tube. Next, $2 \mathrm{~mL}$ of serum was separated by centrifugation $(3,000 \mathrm{r} / \mathrm{min}$, $10 \mathrm{~min}$ ), and the motilin (MTL) and substance P (SP) indexes were detected by commercial ELISA kits (Biolegend, San Diego, USA). The procedure was conducted strictly in accordance with the kit instructions.

\section{Determination of ATP in jejunal tissue by colorimetry}

A $0.5 \mathrm{~cm} \times 0.5 \mathrm{~cm}$ section of rat jejunal tissue was taken and 
washed with physiological saline. The ATP content of rat jejunal epithelial cells were determined by a colorimetric method according to the kit instructions.

\section{Immunobistochemistry}

Myosin light chain kinase (MLCK) expression level was analyzed by immunohistochemistry. Rat paraffin colon sections were routinely fixed with $4 \%$ paraformaldehyde for $10 \mathrm{~min}$ : after rinsing 3 times ( $3 \mathrm{~min} / \mathrm{time}$ ) by phosphate buffered saline, $50 \mu \mathrm{L}$ of peroxidase blocking solution was added dropwise and incubated for $10 \mathrm{~min}$ at room temperature. Slides were then incubated with anti-MLCK primary antibody (Proteintech, IL, USA) overnight at $4{ }^{\circ} \mathrm{C}$, followed by HRP-conjugated anti-rabbit IgG incubation at room temperature for $30 \mathrm{~min}$, and diaminobenzidine (DAB) was used for staining. Image-Pro Plus 6 graphic processing software analysis and high-power microscopy $(\times 200)$ observed the expression of MLCK per unit area of colon tissue, and 5 fields of each section were randomly observed. The respective optical density values of MLCK expression in each group of rats were statistically determined.

\section{Plasma sample preparation for UPLC-Q/TOF-MS analysis}

The rat plasma samples stored in the freezer at $-80{ }^{\circ} \mathrm{C}$ were thawed at $4{ }^{\circ} \mathrm{C}$. Each $200 \mu \mathrm{L}$ plasma sample was treated with 8 volumes of methanol (chromatography grade). The samples were mixed by vortex for $30 \mathrm{~s}$. Then, the mixture was centrifuged at $12,000 \mathrm{r} / \mathrm{min}$ at $4{ }^{\circ} \mathrm{C}$ for $10 \mathrm{~min}$. The supernatant was absorbed with a $1 \mathrm{~mL}$ disposable syringe and passed through the $0.22 \mu \mathrm{m}$ microporous filter membrane, which was the spare sample for further UPLC-Q/TOF-MS analysis. The quality control (QC) sample was prepared by mixing $10 \mu \mathrm{L}$ of each spare sample to be tested.

\section{Mass spectrum analysis and verification of methodology}

To guarantee system repeatability and stability, QC samples were used for method validation. The QC specimen was continuously analyzed 6 times. Chromatographic separation was performed on a Shimadzu UPLC system (Kyoto, Japan) combined with an LC-30AD binary liquid pump, SIL-30SD autosampler, DGU-20A5R on-line solvent degasser, CTO-30A column oven, AB SCIEX Triple TOF 5600+ system, and ESI source. An Agilent C18 reversedphase column $(2.1 \times 100 \mathrm{~mm}, 1.8 \mu \mathrm{m}$, Palo Alto, CA, USA $)$ was applied for all analyses at $35^{\circ} \mathrm{C}$. The mobile phase was composed of $0.1 \%$ formic acid aqueous solution (solvent A, Merck, USA) and acetonitrile (solvent B, Merck, USA) at a flow rate of $0.3 \mathrm{~mL} / \mathrm{min}$. The injection volume was set as $1 \mu \mathrm{L}$. The solvent gradient was as follows: $0-3 \mathrm{~min}$, $5-60 \%$ B; 3-12 min, $60-65 \%$ B; $12-13 \mathrm{~min}, 65-95 \% \mathrm{~B}$; 13-15 min, $95-5 \%$ B. Mass spectrometry experiments were conducted using the Q-TOF MS system equipped with an electrospray ionization (ESI) source in both positive and negative modes. Specific conditions were as follows: ion spray voltage, $+4,500 /-4,500$; source temperature, $550^{\circ} \mathrm{C}$; desolvation gas flow rate, $50 \mathrm{~mL} / \mathrm{min}$; collision energy, $+35 /-35 \mathrm{eV}$; and decluster voltage, $+60 /-60 \mathrm{~V}$. All reagents were of HPLC grade.

\section{Data processing and statistical analysis}

For metabolomics data processing, raw data files were identified and extracted, graded and corrected by Analyst TF 1.6 software (AB SCIEX, Boston, MA, USA). Raw mass spectrometric data were preliminarily managed by Marker View 1.2.1 (AB SCIEX, Boston, MA, USA), the peak area of the chromatographic peak of the compound was standardized by MetaboAnalyst 4.0 software, and the compounds with significant differences were screened out $(\mathrm{P}<0.05$ and multiple change $\mathrm{P}<0.05)$. The resulting data matrices were then introduced into SIMCA (version 14.1, Umetrics, Sweden) for principal component analysis (PCA), and partial least square discriminant analysis (PLS-DA). Orthogonal Projections to Latent Structures Discriminant Analysis (OPLS-DA) was engaged to filter endogenous biomarkers between different groups (15). MassLynx (Waters Technologies, United States) combined with the HMDB database identified the potential biomarkers. Variable importance in projection (VIP) represents the contribution degree of variables in different groups (16). Those with the VIP value $>1.0$ and $\mathrm{P}$ value $<0.05$ among the three groups were estimated by $t$-test or the Mann-Whitney $U$ test. One-way analysis of variance (ANOVA) was engaged by Prism software (GraphPad Software 8.0, San Diego, CA, USA) for assessment of the related pharmacological indicators. The results were considered significant with $\mathrm{P}<0.05$.

\section{Results}

Effect of ZSBZ on feeding behavior and fecal excretion of loperamide-induced constipation rats

To investigate whether treatment with the mixture of ZS 
A

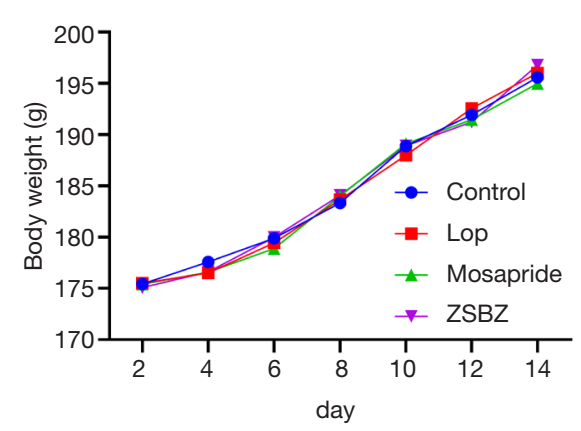

D

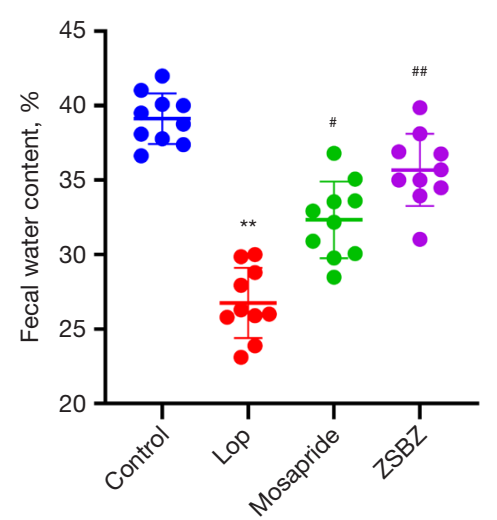

B

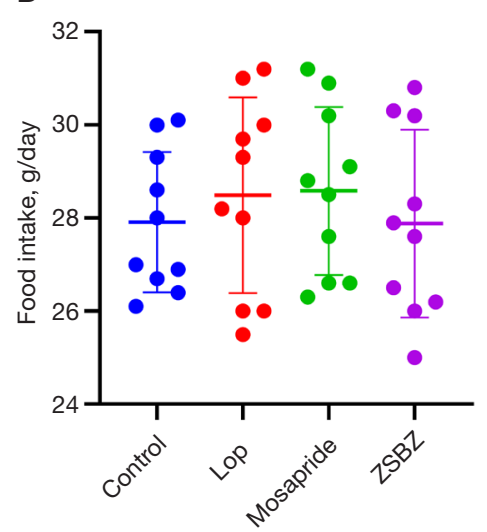

$E$

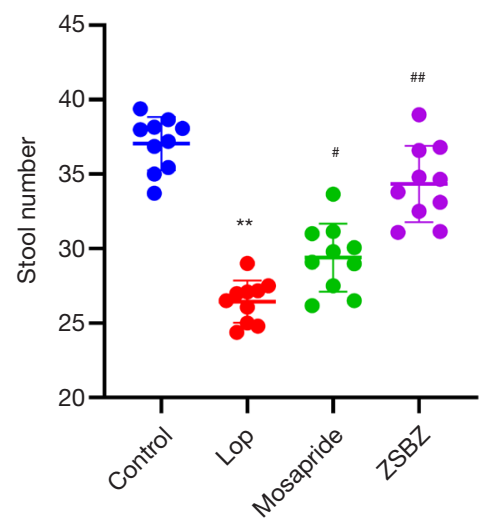

C

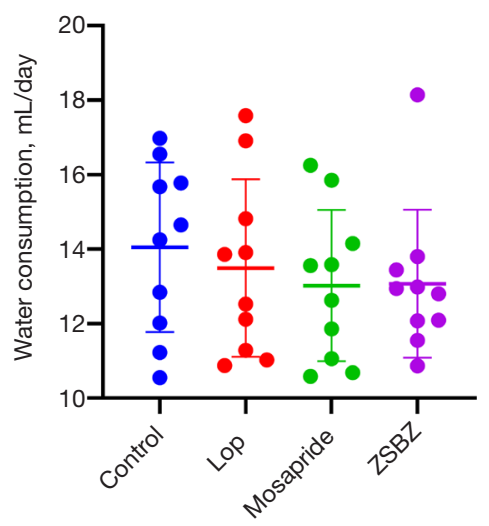

$\mathrm{F}$

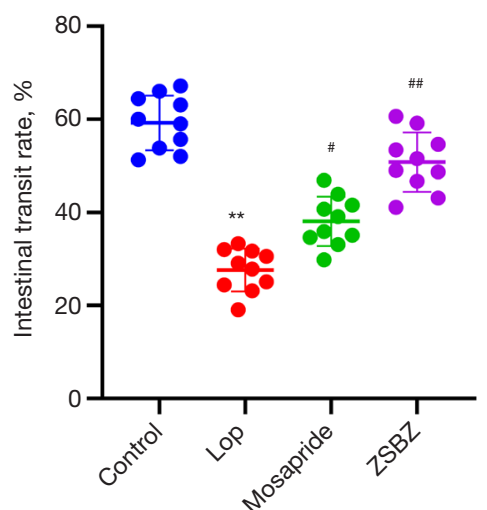

Figure 1 Effects of ZSBZ on the feeding behavior, fecal excretion, and intestinal transit rate in normal and loperamide-induced rats. Data represent the mean $\pm \mathrm{SD}(\mathrm{n}=10)$. Significant levels were marked with strikes presented as compared with the control group (control): ${ }^{* *} \mathrm{P}<0.01$, compared with the Lop group: ${ }^{\#} \mathrm{P}<0.05,{ }^{\# \#} \mathrm{P}<0.01$.

and BZ could affect body weight, feeding behavior and fecal excretion in constipated rats, changes in body weight, food intake, water consumption, fecal water content and stool number were measured during the experimental period. As shown in Figure 1, body weight steadily increased in all groups (Figure 1A). However, no significant differences in body weight, food intake and water consumption were observed among any groups after administration of loperamide, mosapride and the $\mathrm{ZS}$ and $\mathrm{BZ}$ mixture (Figure $1 A, B, C$ ). Fecal water content was significantly decreased after treatment with loperamide compared with that in the control group $(\mathrm{P}<0.01)$. Otherwise, the $\mathrm{ZS}$ and $\mathrm{BZ}$ mixture treatment increased the fecal water content compared with the Lop treatment (Figure 1D). Moreover, rats with constipation exhibited a decreased stool number, while those in the mosapride- and ZS and BZ mixture-treated groups showed improved differences (Figure 1E). Collectively, these results indicate that the $\mathrm{ZS}$ and $\mathrm{BZ}$ mixture treatment did not induce any alteration of body weight or feeding behavior and exerted effects on fecal water content and stool number.

\section{Effect of ZSBZ treatment on gastrointestinal motility}

As shown in Figure 1F, significant decreases in the intestinal transit ratio of charcoal were observed in the Lop group compared with the control group. After 2 weeks, the intestinal transit rate in the $\mathrm{ZS}$ and $\mathrm{BZ}$ mixture group was significantly higher than that in the Lop group and lower than that in the control group. Similar results were found for mosapride treatment. 

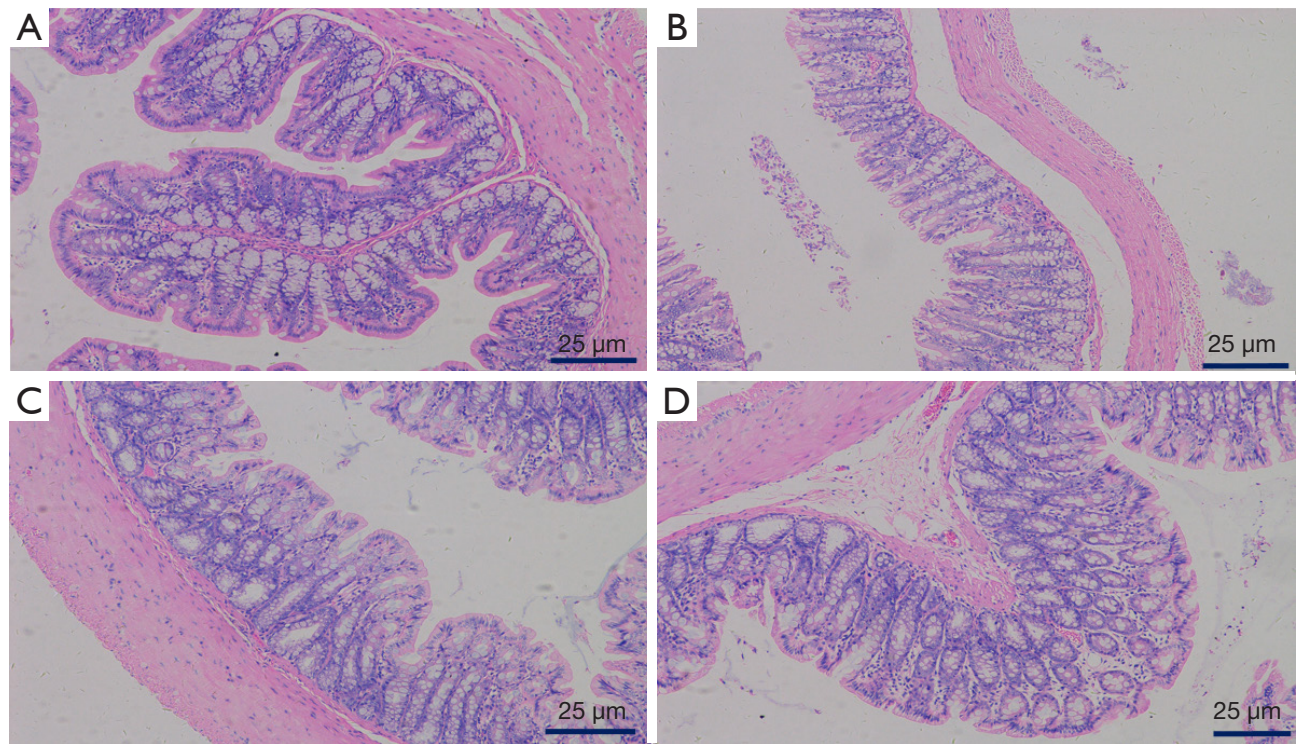

Figure 2 Histological structures of colon in Lop-induced constipation rat model after ZSBZ treatment. H\&E stained sections of colon from the control (A), Lop (B), mosapride (C) and ZSBZ (D) treated groups were observed at 400× magnification using a light microscope. The change of smooth muscle cells, neuron cells, and inflammatory cells were observed at 200x.

\section{Histological alteration of colon}

To investigate the effect of the ZSBZ herb pair on structural alteration of the colon tissue, the smooth muscle cell, neuronal cell, and mucous layer in the colon were detected by H\&E staining. In the Lop group, the colonic smooth muscle cells of the rats were atrophied and arranged in a disorderly fashion, the neuronal cells in the colon tissue were reduced, and the inflammatory cells infiltrated the mucosa. However, the colonic smooth muscle cells and neuronal cells of the ZSBZ group gradually recovered to the same level as those in the control group, and the infiltration of inflammatory cells in the colon tissue was reduced (Figure 2).

\section{Effects of ZSBZ on serum motilin (MTL), substance P (SP) and adenosine triphosphate (ATP) in rats}

The effects of ZSBZ on relieving constipation were further evaluated by measuring serum neurotransmitters (MTL and SP) and ATP in jejunal tissue. As shown in Figure 3, the levels of MTL, SP and ATP were significantly lower in constipated rats than in control rats $(\mathrm{P}<0.05)$. In comparison to the Lop group, the ZSBZ group showed significant increases in the levels of MTL, SP and ATP $(\mathrm{P}<0.01)$. These results demonstrate that $\mathrm{ZS}$ and $\mathrm{BZ}$ mixture treatment effectively increased the levels of the excitatory neurotransmitters (MTL and SP) and adenosine triphosphate to speed the motility of the gastrointestinal tract.

\section{Effect on MLCK expression in colon tissues in rats}

It can be observed in Figure 4 that brownish yellow granules in the cytoplasm of smooth muscle cells were found in the mucosa, submucosa and muscular layer of colon tissue of rats in each group, and brownish yellow granules with different shades and densities can be seen. Compared with that in the control group, the cytoplasmic staining of the smooth muscle cells in the muscular layer of the colon of the Lop group was light yellow, and the optical density of MLCK was significantly reduced $(\mathrm{P}<0.01)$. Compared with that in the Lop group, the optical density of MLCK in the ZSBZ group was significantly increased $(\mathrm{P}<0.05)$.

\section{Visual inspection of $Q C$ overlapping total ion chromatography}

The overlapping total ion chromatograms (TIC) are presented in Figure $5 A, B$. In the common modes of positive and negative ions, the QC samples gather near the middle of the projection of the score matrix, and the significantly overlapping chromatogram indicated that the UPLC-Q/ TOF-MS system was repeatable and stable throughout the 

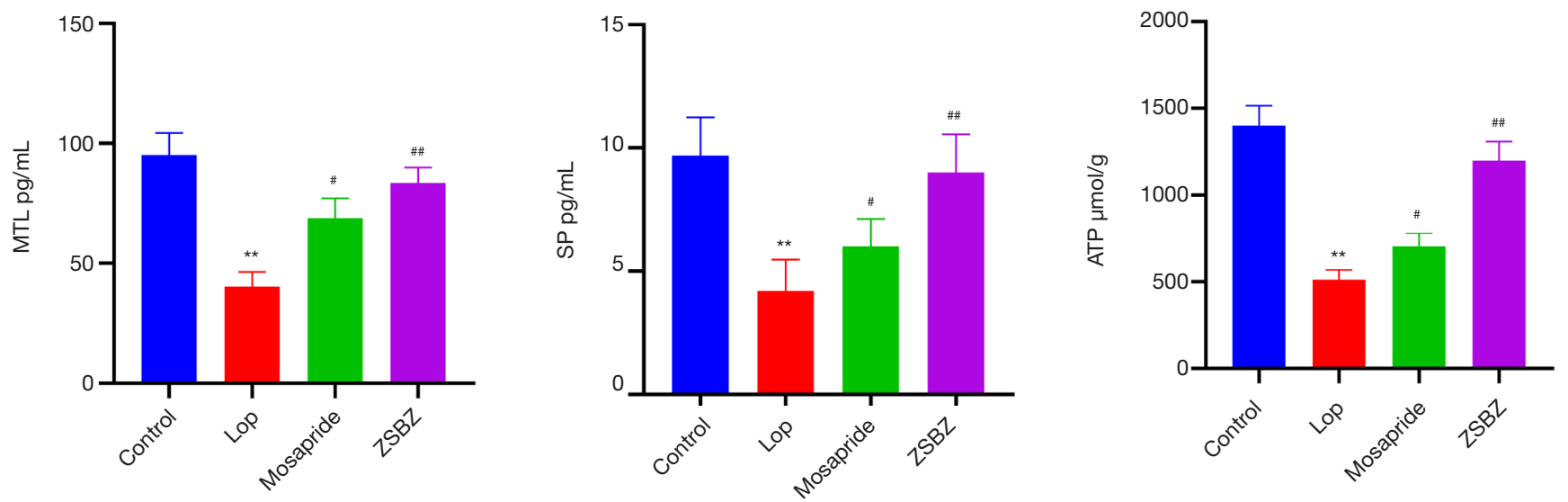

Figure 3 Effects of ZSBZ on serum parameters and adenosine triphosphate in rats. Data represent the mean \pm SD ( $n=10$ ). Significant levels were marked with strikes presented as compared with the control group (control): ${ }^{* *} \mathrm{P}<0.01$, compared with the Lop group: ${ }^{\#} \mathrm{P}<0.05$, ${ }^{\# \#} \mathrm{P}<0.01$.
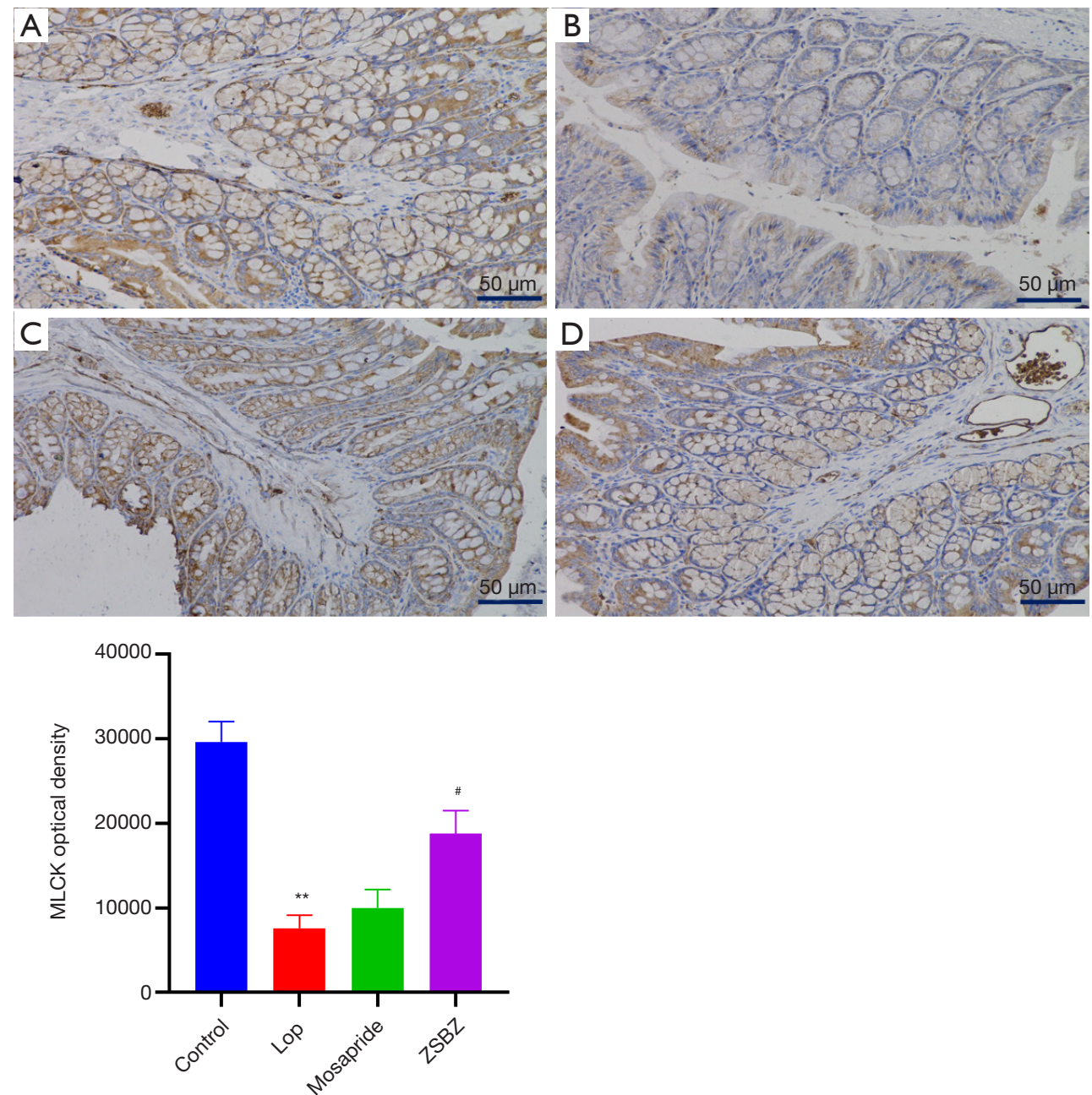

Figure 4 The expression of myosin light chain kinase (MLCK) in colon on protein level via immunohistochemistry. Significant levels were marked with strikes presented as compared with the control group (control): ${ }^{* *} \mathrm{P}<0.01$, compared with the Lop group: ${ }^{*} \mathrm{P}<0.05$. 

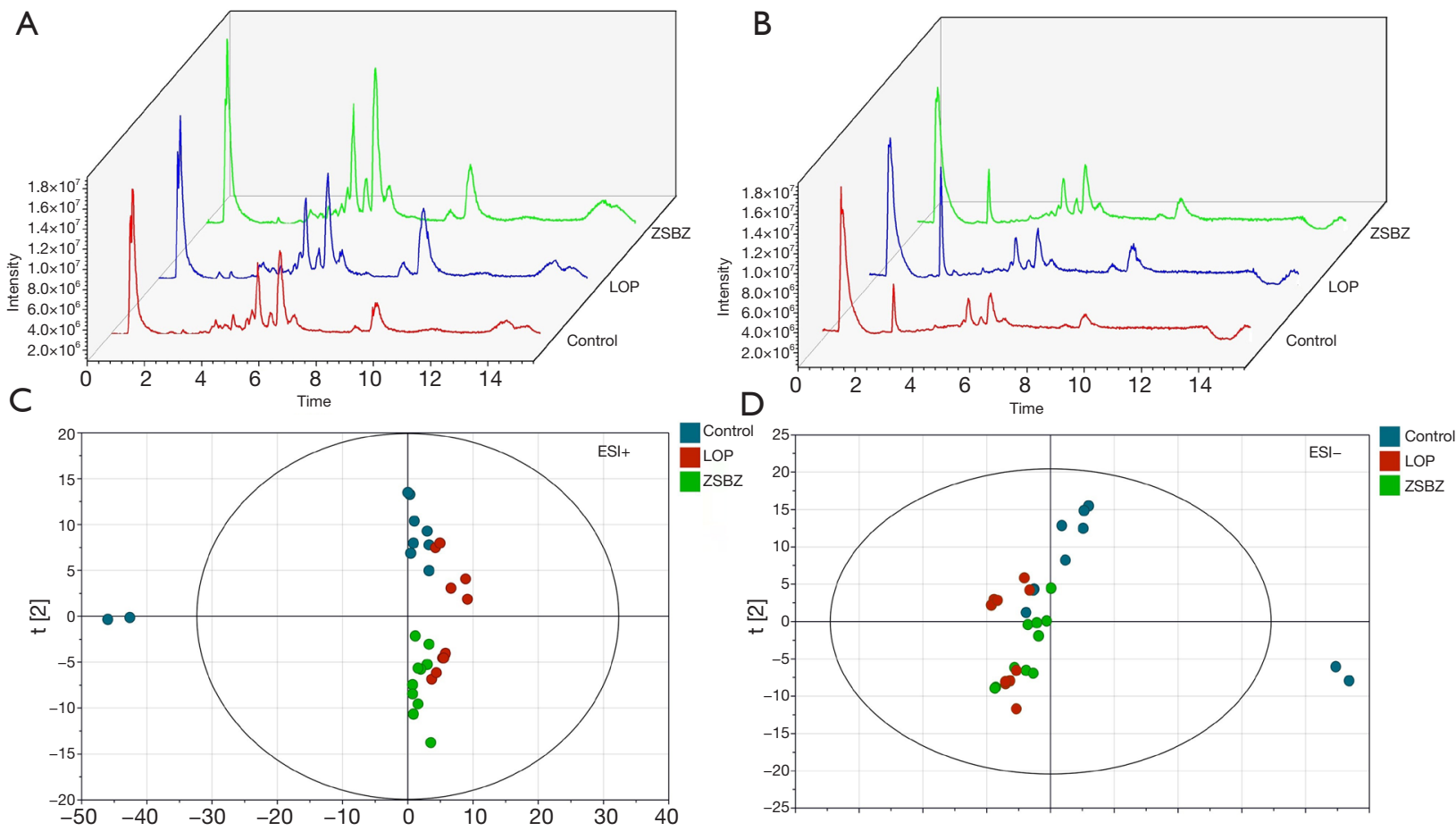

Figure 5 UPLC-Q/TOF-MS total ion chromatograms (TIC) of plasma samples among control, Lop and ZSBZ groups in ESI ${ }^{+}$mode (A) and ESI- mode (B). Effect of ZSBZ on the metabolic profiling by PCA score plots. PCA score plot of positive ion mode (C) and negative ion mode (D) for the control, Lop and ZSBZ groups.

entire analysis process.

\section{Multivariate statistical analysis of UPLC-Q/TOF-MS}

The UPLC-Q/TOF-MS data were analyzed using PCA to determine the differences between the principal components of the control group, Lop group and ZSBZ group. PCA score plots are shown in Figure 5C,D. The PCA model was estimated by cross-validation in the positive mode $\left(\mathrm{R}^{2} \mathrm{X}=0.685, \mathrm{Q}^{2}=0.534\right)$ and in the negative mode $\left(\mathrm{R}^{2} \mathrm{X}=0.698\right.$ and $\left.\mathrm{Q}^{2}=0.468\right)$, respectively. Orthogonal projections to latent structures discriminant analysis (OPLS-DA) was applied to screen the potential markers formed by the metabolic differences among the groups. Figure $6 A, B, C, D$ shows a certain extent of separation tendency among the control, Lop and ZSBZ groups, indicating that the physiological and metabolic environments of metabolites in the plasma of constipated rats were changed after ZSBZ treatment. Since the values of both $\mathrm{R}^{2} \mathrm{X}$ and $\mathrm{Q}^{2}$ approach 1.0 (Table 1), we conclude that the accuracy and predictive capability of the models were excellent.

\section{Identification of the different metabolites in the plasma samples among different groups}

Based on the establishment of the OPLS-DA model, the differences among variables between control group and Lop group, and Lop group and ZSBZ group, were screened by volcano plot analysis index (Figure $6 E, F, G, H$ ). The complete data set was imported into MetaboAnalyst 4.0 to generate a heatmap to identify group differences (Figure 7). According to the variable projection importance index (VIP) of each OPLS-DA model, the VIP value was positively correlated with the grouping contribution rate. The variables ( $\mathrm{m} / \mathrm{z}$-Rt pairs) that contributed to the distinction were filtered according to variable importance in projection (VIP) value $>1.0$ of the OPLS-DA model and t-test $\mathrm{P}$ value $<0.05$. The secondary mass spectra of different substances were extracted by PeakView software provided by AB SCIEX company, then compared with the standard secondary mass spectrometry data from MassBank, Chemspider, HMDB, METLIN and other compound databases and related literature. Finally, 9 differential metabolites of each group, including L-proline, cis-aconitic acid, L-arginine, 

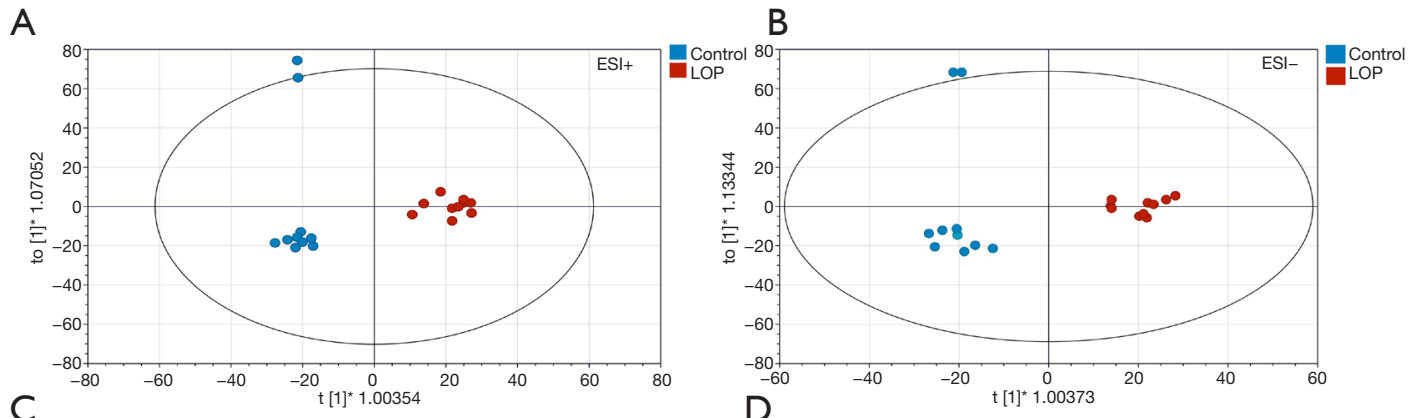

C
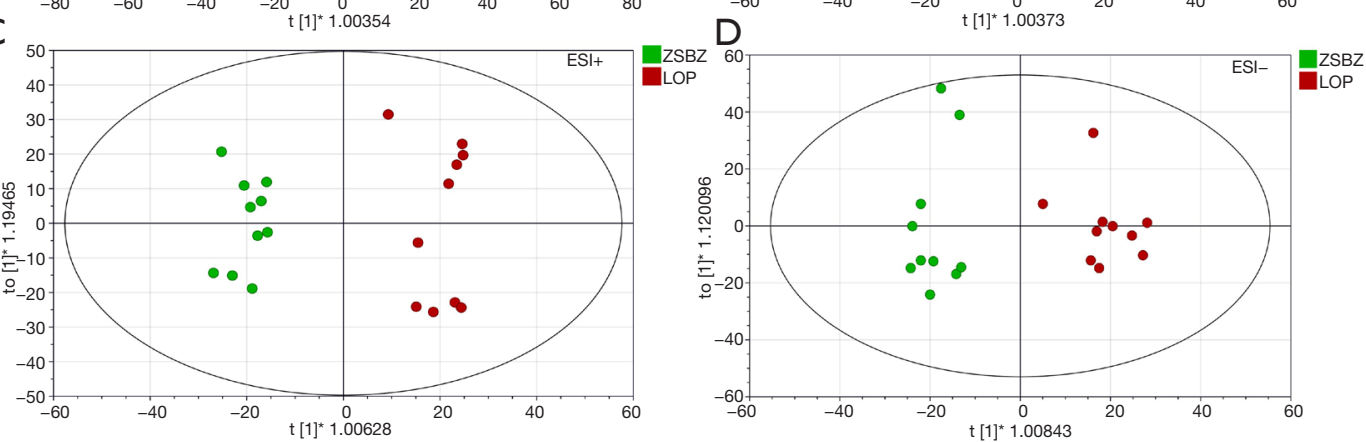

$\mathrm{E}$
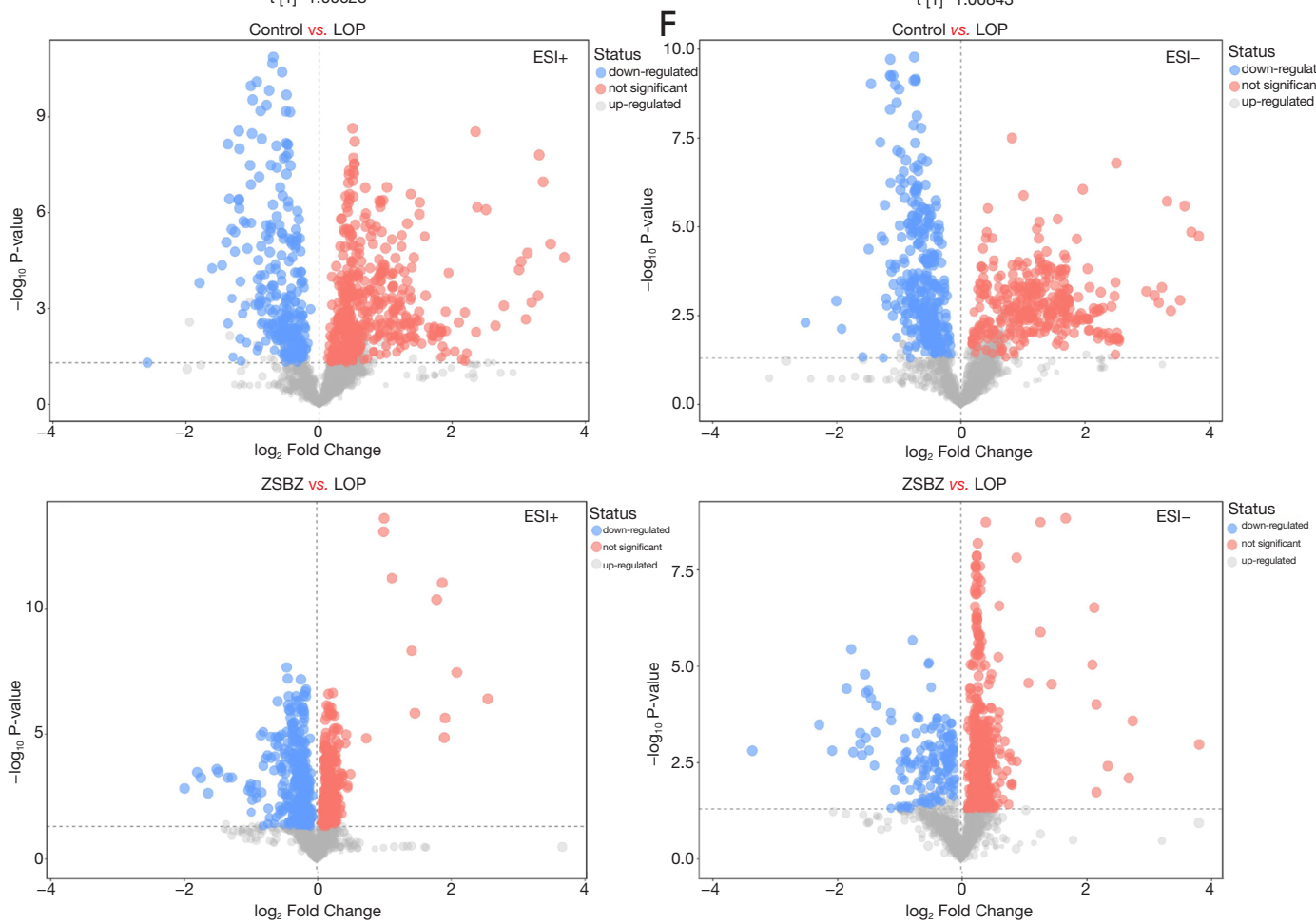

Figure 6 The score plots from OPLS-DA model and volcanic map based on metabolic profiling. (A,C) OPLS-DA score plot of positive ion mode. (B,D) OPLS-DA score plot of negative ion mode. OPLS-DA score plot of ZSBZ/LOP group in positive (C) and (D) negative ion mode. Volcano plot of Control/LOP group in positive (E) and (F) negative ion mode. Volcano plot of ZSBZ/LOP group in positive (G) and (H) negative ion mode. ESI+ means the positive electrospray ionization. ESI- means the negative electrospray ionization. 
Table 1 Summary of parameters for PCA and OPLS-DA models

\begin{tabular}{|c|c|c|c|c|c|c|c|}
\hline No. & Model & \multicolumn{3}{|c|}{ ESI+ } & \multicolumn{3}{|c|}{ ESI- } \\
\hline M1 & PCA & 0.685 & - & 0.534 & 0.698 & - & 0.468 \\
\hline M2 & OPLS-DA & 0.573 & 0.962 & 0.906 & 0.613 & 0.955 & 0.894 \\
\hline M3 & OPLS-DA & 0.681 & 0.954 & 0.863 & 0.738 & 0.927 & 0.821 \\
\hline
\end{tabular}

Note: M1 represents the PCA model of metabolic profiling of control, Lop and ZSBZ groups; M2 represents the OPLS-DA model of metabolic profiling of control and Lop groups; M3 represents the OPLS-DA model result of metabolic profiling of Lop and ZSBZ groups.

paraxanthine, isocitric acid, pyridoxal 5'-phosphate, gammalinolenic acid, cholic acid and LysoPC [22:5 (4Z, 7Z, 10Z, $13 Z, 16 Z)]$, were identified by comprehensive analysis: the results are shown in Table 2. Furthermore, the relative contents of 9 differential metabolites in rats in each group were tested by nonpaired t-test, and the results are shown in Figure 8. The results showed that the administration of ZSBZ could significantly improve the abnormal changes in 9 differential metabolites in the Lop group.

\section{Metabolomics pathway analysis}

We input the above identified endogenous differential metabolites into the MetaboAnalyst 4.0 metabonomics online analysis platform (https://www.metaboanalyst.ca/faces/home. xhtml), selected the Rattus norvegicus (rat) pathway library as the metabolic pathway database, set the hypergeometric test as pathway enrichment analysis, with relative-betweenness centrality pathway analysis topology, carried out pathway enrichment, and screened out metabolic pathways with a metabolic pathway influence value (Impact) greater than 0.10 (Table 3). Plots were described by pathway impact scores ( $\mathrm{x}$-axis) and $\mathrm{P}$ values (y-axis). The values of pathway impact and $-\log \mathrm{P}$ were larger, the correlation of the metabolic differences between different groups was higher, and the circle in Figure 9 became larger and redder. Plots were described by pathway impact scores ( $\mathrm{x}$-axis) and $\mathrm{P}$ values (y-axis). In this study, the pathway impact-value was set to 0.10 according to reference (17). The results of pathway analysis are shown in Figure 9. The results showed that the main metabolic pathways involving differential metabolites in plasma samples of each group included caffeine metabolism, vitamin B6 metabolism, arginine and proline metabolism and the citrate cycle (TCA cycle). It is suggested that the metabolic abnormality of rats with slow transit constipation and the therapeutic effects of Fructus Aurantii and Atractylodes macrocephala on slow transit constipation are closely related to the above metabolic pathways.

\section{Discussion}

In this study, a UPLC-Q/TOF-MS-based metabolomics approach was implemented to explore the potential mechanisms of ZSBZ in the treatment of constipation. The method used in this experiment is not to extract a single chemical component but to highlight the integrity of Chinese medicine, which is more in line with the synergistic mechanism of Chinese medicine compound prescription as a whole. We found that ZSBZ treatment decreased the intestinal propulsion rate in constipated rats. To support our result, previous findings have shown that ZSBZ improved the intestinal motility in constipated rats by using the charcoal transit ratio (18).

MTL is a very important gastrointestinal hormone that can promote gastrointestinal motility and enhance gastrointestinal contractility and tension (19). SP is a neurotransmitter that has the function of stimulating gastrointestinal motility, is widely distributed throughout the entire gastrointestinal tract and intestinal nervous system, and can stimulate the contraction of almost all digestive tract smooth muscle (20). Both of them can effectively reflect the changes in gastrointestinal function.

After treatment with ZSBZ, the colonic motility disorder and the expression of related gastrointestinal hormones in STC rats were significantly improved. The results show that ZSBZ may regulate two aspects of gastrointestinal function: the gastrointestinal-neuromuscular regulation system and gastrointestinal hormones.

Intestinal movement and nutrition absorption are closely related to adenosine triphosphate (ATP). ATP is the most basic carrier of energy for conversion in organisms, and the change in ATP content is directly related to the energy metabolism of various organs (21). As the most important energy molecule, ATP plays important roles in various physiological and 
A

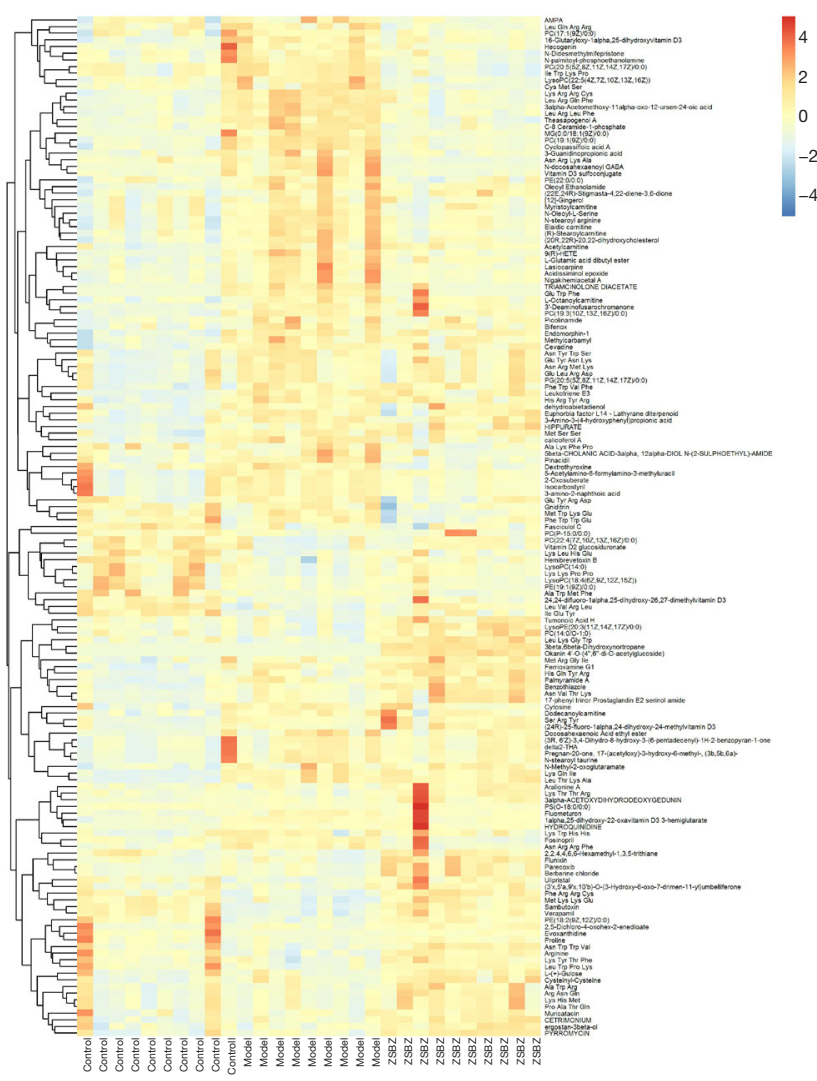

B

ESI-

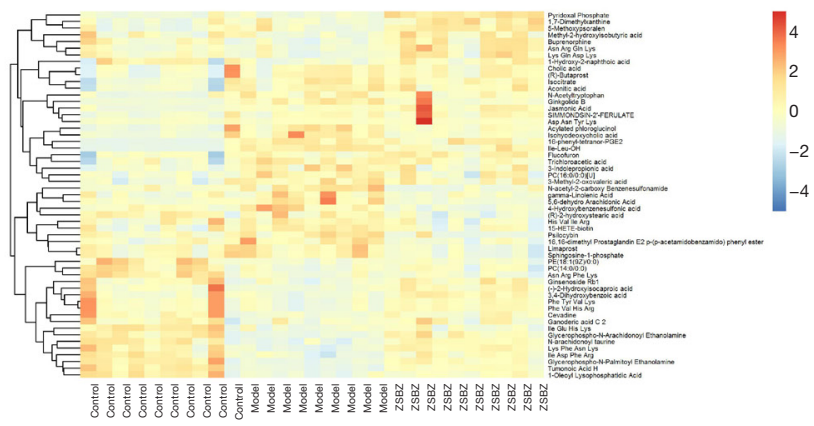

Figure 7 Heat map of differential metabolites. (A) Heat map of 153 differential metabolites in positive ion mode; (B) heat map of 55 differential metabolites in negative ion mode.

Table 2 Potential biomarkers in plasma associated with STC based on the UPLC-Q-TOF/MS analysis

\begin{tabular}{|c|c|c|c|c|c|c|c|c|c|}
\hline No. & Metabolites & $\mathrm{m} / \mathrm{z}$ & $\operatorname{Rt}(\mathrm{s})$ & HMDB & Mode & VIP & P-VALUE & $\mathrm{M} / \mathrm{NC}$ & $\mathrm{M} / \mathrm{ZSBZ}$ \\
\hline 2 & cis-Aconitic acid & 173.01 & 67.034 & HMDB0000072 & - & 1.10 & 0.0011 & $\uparrow$ & $\downarrow$ \\
\hline 3 & L-Arginine & 175.12 & 46.831 & HMDB0000517 & + & 1.30 & 0.0104 & $\downarrow$ & $\uparrow$ \\
\hline 4 & Paraxanthine & 179.05 & 45.5845 & HMDB0001860 & - & 1.31 & 0.0045 & $\uparrow$ & $\downarrow$ \\
\hline 6 & Pyridoxal 5'-phosphate & 246.02 & 151.181 & HMDB0001491 & - & 1.73 & 0.0000 & $\downarrow$ & $\uparrow$ \\
\hline 7 & Gamma-Linolenic acid & 277.22 & 863.0905 & HMDB0003073 & - & 1.19 & 0.0324 & $\uparrow$ & $\downarrow$ \\
\hline 8 & Cholic acid & 407.28 & 239.492 & HMDB0000619 & - & 1.07 & 0.0097 & $\uparrow$ & $\downarrow$ \\
\hline 9 & LysoPC [22:5 (4Z,7Z,10Z,13Z,16Z)] & 570.35 & 327.3155 & HMDB0010402 & + & 1.16 & 0.0017 & $\uparrow$ & $\downarrow$ \\
\hline
\end{tabular}

VIP: variable importance in the projection, was obtained from the PLS-DA mode. P value was calculated by t-test or Mann-Whitney $U$ test. 

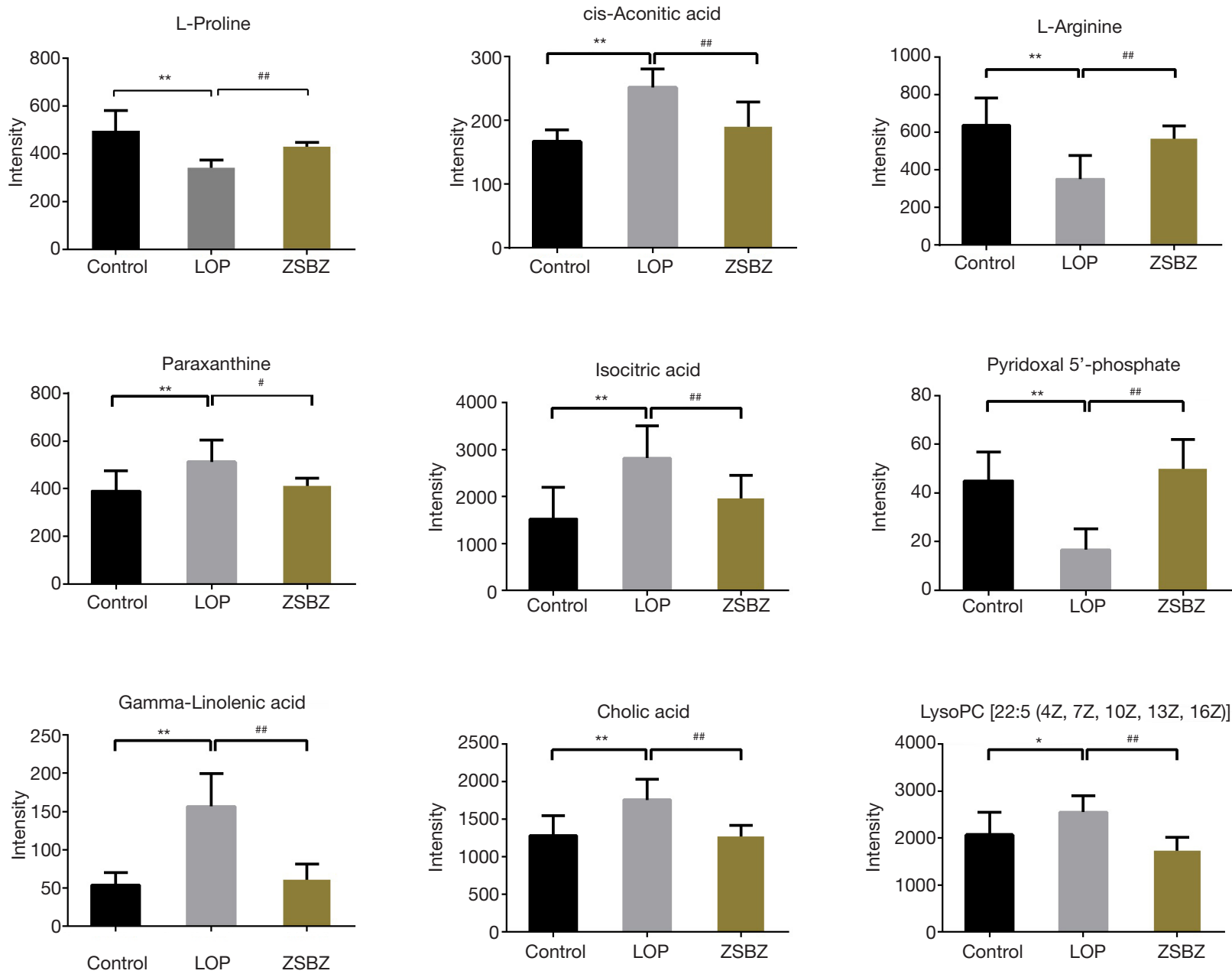

Figure 8 Comparison of potential biomarkers in three groups after treatment. ${ }^{*} \mathrm{P}<0.05,{ }^{* *} \mathrm{P}<0.01$ compared with control group; ${ }^{\#} \mathrm{P}<0.05$, ${ }^{\#} \mathrm{P}<0.01$ compared with Lop group.

Table 3 Pathway analysis result with MetaboAnalyst 4.0

\begin{tabular}{|c|c|c|c|c|}
\hline Pathway name & Match status & Raw $p$ & $-\log (p)$ & Impact \\
\hline Vitamin B6 metabolism & $1 / 9$ & 0.13994 & 1.9665 & 0.2549 \\
\hline Arginine and proline metabolism & $2 / 38$ & 0.12898 & 2.0481 & 0.13566 \\
\hline Citrate cycle (TCA cycle) & $2 / 20$ & 0.041728 & 3.1766 & 0.09501 \\
\hline Glyoxylate and dicarboxylate metabolism & $2 / 32$ & 0.096588 & 2.3373 & 0.02381 \\
\hline Glycerophospholipid metabolism & $1 / 36$ & 0.45587 & 0.78555 & 0.01736 \\
\hline
\end{tabular}

The Match Status in the table is all the endogenous metabolites contained in the pathway and the number of metabolites found in this pathway. Raw $\mathrm{p}$ is the original $\mathrm{P}$ value obtained by the analysis pathway. Impact refers to the influence value of the pathway, which is obtained by topological analysis. 


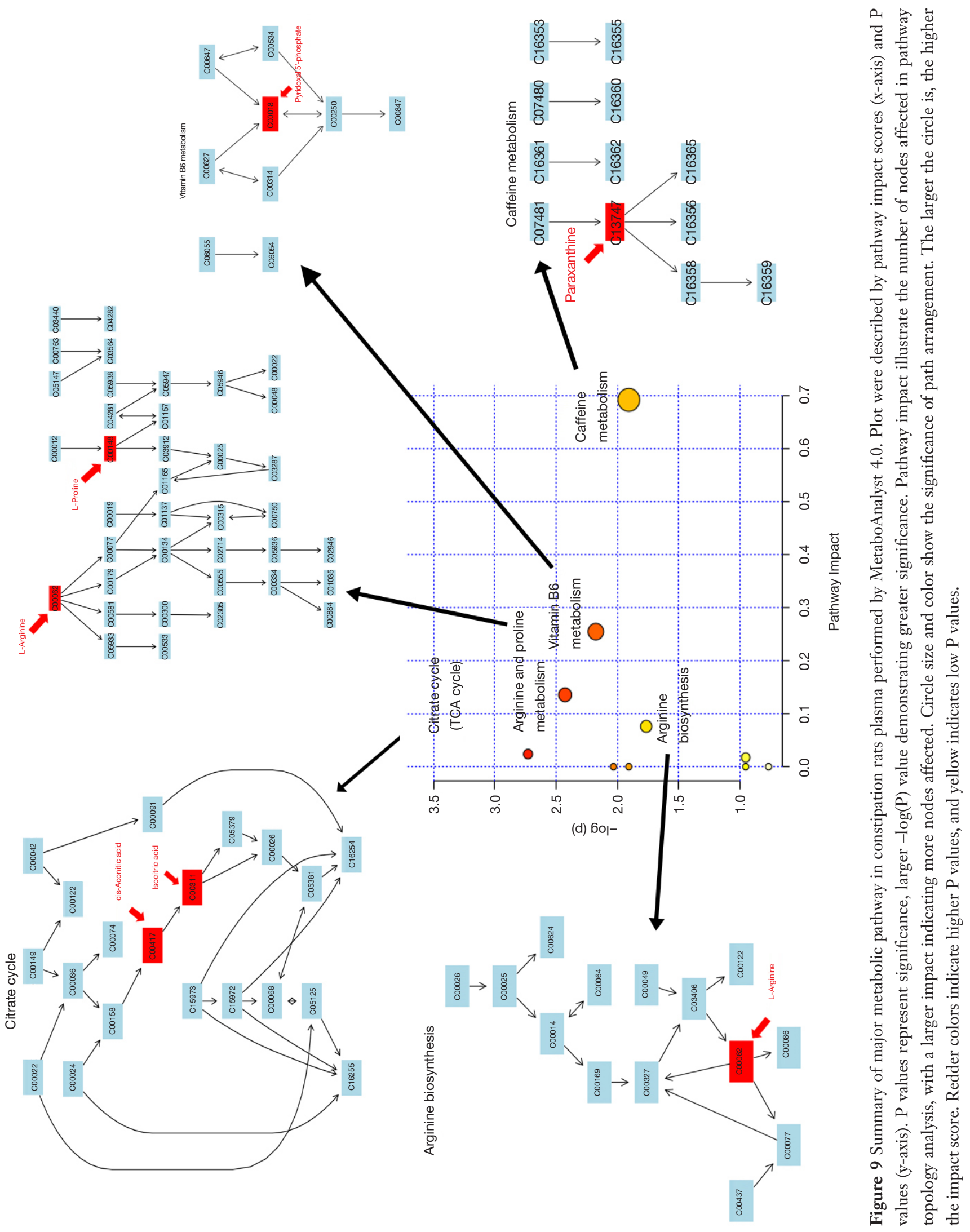


pathological processes of cells. The results showed that the colonic motor function of STC rats decreased significantly, which led to the decrease in ATP production. After the treatment with ZSBZ, the colonic motor function of STC model rats was significantly enhanced, which was reflected in the significant increase in ATP content.

Studies have shown that smooth muscle contraction plays an important role in regulating the changes in gastrointestinal motility, and the decrease in contractility will lead to the decrease in intestinal peristalsis, which in turn leads to constipation (22).

MLCK is the key protein for smooth muscle contraction: when smooth muscle cells are stimulated by electrical signals, endoplasmic reticulum and extracellular calcium ions are released into the cytoplasm, intracellular calcium concentration increases, and $\mathrm{Ca}^{2+}$ forms a complex with CaM, which further activates MLCK to further phosphorylate myosin light chain (MLC), thus causing myosin and actin to slide relative to each other, and ultimately causing smooth muscle cells to contract (23). Immunohistochemical analysis demonstrated that the expression of MLCK in colonic smooth muscle cells of STC rats was significantly lower than that of normal rats, while ZSBZ could significantly increase the expression of MLCK in colonic smooth muscle cells of STC rats, suggesting that the regulation of the signal transduction mechanism of $\mathrm{Ca}^{2+}-\mathrm{CaM}-\mathrm{MLCK}$ in colonic smooth muscle cells may be one of the most important mechanisms of ZSBZ with respect to the regulation of colonic motility.

Metabonomic results showed that slow transit constipation could lead to an abnormal increase in p-xanthine, which could be decreased after administration of ZSBZ. Paraxanthine is a very weak polar compound which participates in a variety of enzymatic reactions in vivo: in particular, p-xanthine and formaldehyde can be biosynthesized from caffeine, which is catalyzed by the cytochrome P450 1A2 enzyme. In addition, paraxanthine and acetyl-CoA can be converted into 5-acetylamino-6formylamino-3-methyluracil through interaction with the arylamine $\mathrm{N}$-acetyltransferase 2 enzyme. In humans, paraxanthine is involved in caffeine metabolism. The effect of p-xanthine on constipation has not been reported. Studies on caffeine and colorectal cancer are more common. Some studies have shown that caffeine can reduce the inflammatory process by changing the balance between proinflammatory and anti-inflammatory cytokines, thus inhibiting the progression of colorectal cancer (24).

Pyridoxal 5'-phosphate participates in vitamin B6 metabolism. Studies have shown that abnormal levels of vitamin B6 are closely related to constipation (25). In addition, vitamin B6 is involved in the formation of serotonin, which increases the concentration of serotonin. In addition, vitamin B6, as a coenzyme, is involved in various metabolic reactions, such as those of amino acids and proteins. Lack of vitamin B6 can cause protein hydrolysis to stagnate and result in conversion into fat, which prevents growth. Administration of ZSBZ can increase the content of pyridoxal 5'-phosphate, thus increasing the synthesis of vitamin B6, increasing the content of serotonin and promoting the metabolism of protein (26).

Cis-aconitic acid and isocitric acid participate in the tricarboxylic acid cycle. The tricarboxylic acid cycle is a common metabolic pathway for three major classes of nutrients (sugars, lipids and amino acids) in the human body and other aerobic organisms. In the tricarboxylic acid cycle, isocitrate can be catalyzed by isocitrate dehydrogenase, which is irreversible and is also the rate-limiting step in the tricarboxylic acid cycle (27). Compared with the model group, ZSBZ significantly decreased the contents of cis-aconitic acid and isocitric acid in the blood of rats, indicating that ZSBZ may promote tricarboxylic acid circulation, which is conducive to the processes of glycolysis and fat mobilization, so that it can quickly provide a portion of the energy for the contraction of large intestinal smooth muscle and promote the movement of the large intestine. Therefore, regulating tricarboxylic acid cycle metabolism is probably associated with the therapeutic effect of ZSBZ in constipation. Additionally, evidence has indicated that the tricarboxylic acid cycle was involved in the abnormal metabolism of colitis (28).

L-Arginine and L-proline participate in the metabolic pathways of proline and arginine. $\mathrm{L}$-arginine (Arg) is a semiessential amino acid with many physiological functions. Studies have shown that arginine and its downstream metabolite proline can increase the diversity of intestinal flora in mice, thus fulfilling a protective role in intestinal inflammation (29). In this study, levels of L-arginine and L-proline were remarkably upregulated after ZSBZ treatment in constipated rats, making L-arginine and L-proline possible targets for the treatment of constipation. In addition to the above metabolic pathways, the ameliorative effect of ZSBZ on slow transit constipation is also related to arginine biosynthesis, glyoxylate and dicarboxylate metabolism and glycerophospholipid metabolism. These findings further indicate that the metabolic markers could be considered potential candidates 
for the treatment of constipation, although more clinical samples and pharmacological experiments are required to confirm the mechanism of ZSBZ.

\section{Conclusions}

In conclusion, we demonstrated that ZSBZ treatment exhibited a motivating effect on loperamide-induced constipation in rats, the effects of which might be due to the regulation of caffeine and vitamin B6 metabolism. A major challenge for the future is characterizing the individual functions of the altered metabolites circulating in blood to elucidate the underlying mechanisms of ZSBZ on constipation. Based on these metabolomics results, we are able to obtain a broad understanding of the therapeutic effects of ZSBZ. Therefore, the present study will provide additional insight into clinical applications and pharmacological research, as well as the identification of metabolites of ZSBZ in the treatment of constipation patients.

\section{Acknowledgments}

Funding: Supported by China Postdoctoral Science Foundation (2017M620220), the technology project of Jiangsu Provincial Administration of Traditional Chinese Medicine (YB2017061), Scientific Foundation of Integrated traditional Chinese and Western Medicine of Suzhou (SYSD2018212), Young Elite Scientists Sponsorship Program by China Association of Chinese Medicine (CACM-2018-QNRC2-C13), the Open Project Program of Jiangsu Key Laboratory for Pharmacology and Safety Evaluation of Chinese Materia Medica (No. JKLPSE201819), the Project of the Priority Academic Program Development of Jiangsu Higher Education Institutions (PAPD), the fifth batch of Gusu health personnel training project in Suzhou (GSWS2019065) and Suzhou Science and Technology Development Plan people's livelihood Science and Technology - Research on the Application of key Technologies (SS2019073).

\section{Footnote}

Reporting Checklist: The authors have completed the ARRIVE reporting checklist. Available at http://dx.doi. org/10.21037/apm-20-280

Data Sharing Statement: Available at http://dx.doi. org/10.21037/apm-20-280
Conflicts of Interest: All authors have completed the ICMJE uniform disclosure form (available at http://dx.doi. org/10.21037/apm-20-280). The authors have no conflicts of interest to declare.

Ethical Statement: The authors are accountable for all aspects of the work in ensuring that questions related to the accuracy or integrity of any part of the work are appropriately investigated and resolved. The study was approved by ethics board of Suzhou TCM Hospital Affiliated to Nanjing University of Chinese Medicine (NO.2018-03-216) in compliance with national and institutional guidelines for the care and use of animals.

Open Access Statement: This is an Open Access article distributed in accordance with the Creative Commons Attribution-NonCommercial-NoDerivs 4.0 International License (CC BY-NC-ND 4.0), which permits the noncommercial replication and distribution of the article with the strict proviso that no changes or edits are made and the original work is properly cited (including links to both the formal publication through the relevant DOI and the license). See: https://creativecommons.org/licenses/by-nc-nd/4.0/.

\section{References}

1. Polymeros D, Beintaris I, Gaglia A, et al. Partially hydrolyzed guar gum accelerates colonic transit time and improves symptoms in adults with chronic constipation. Dig Dis Sci 2014;59:2207-14.

2. Leung L, Riutta T, Kotecha J, et al. Chronic constipation: an evidence-based review. J Am Board Fam Med 2011;24:436-51.

3. Shahid S, Ramzan Z, Maurer AH, et al. Chronic idiopathic constipation: more than a simple colonic transit disorder. J Clin Gastroenterol 2012;46:150-4.

4. Chen Z, Lin S, Jiang Y, et al. Effects of Bread Yeast Cell Wall Beta-Glucans on Mice with Loperamide-Induced Constipation. J Med Food 2019;22:1009-21.

5. Ouyang A, Locke GR. Overview of neurogastroenterologygastrointestinal motility and functional GI disorders: Classification, prevalence, and epidemiology. Gastroenterol Clin North Am 2007;36:485-98.

6. Bharucha AE, Wald A. Chronic Constipation. Mayo Clin Proc 2019;94:2340-57.

7. Emmanuel A, Cools M, Vandeplassche L, et al. Prucalopride improves bowel function and colonic transit time in patients with chronic constipation: an integrated 
analysis. Am J Gastroenterol 2014;109:887-94.

8. Wang C, Ren Q, Chen XT, et al. System PharmacologyBased Strategy to Decode the Synergistic Mechanism of Zhi-zhu Wan for Functional Dyspepsia. Front Pharmacol 2018;9:841.

9. Zhang X, Li P, Hua Y, et al. Urinary metabolomics study the mechanism of Taohong Siwu Decoction intervention in acute blood stasis model rats based on liquid chromatography coupled to quadrupole time-offlight mass spectrometry. J Chromatogr B Analyt Technol Biomed Life Sci 2018;1074-1075:51-60.

10. Zhuang Y, Qin K, Yu B, et al. A metabolomics research based on UHPLC-ESI-Q-TOF-MS coupled with metabolic pathway analysis: Treatment effects of stir-frying Xanthii Fructus on allergic rhinitis in mice model. Biomed Chromatogr 2018;32:e4352.

11. Liu K, Song Y, Liu Y, et al. An integrated strategy using UPLC-QTOF-MSE and UPLC-QTOF-MRM (enhanced target) for pharmacokinetics study of wine processed Schisandra Chinensis fructus in rats. J Pharm Biomed Anal 2017;139:165-78.

12. Song Y, Su D, Lu T, et al. Differential pharmacokinetics and the brain distribution of morphine and ephedrine constitutional isomers in rats after oral administration with Keke capsule using rapid-resolution LC-MS/MS. J Sep Sci 2014;37:352-9.

13. Su D, Li W, Xu Q, et al. New metabolites of acteoside identified by ultra-performance liquid chromatography/ quadrupole-time-of-flight $\mathrm{MS}(\mathrm{E})$ in rat plasma, urine, and feces. Fitoterapia 2016;112:45-55.

14. Jabri MA, Wannes D, Hajji N, et al. Role of laxative and antioxidant properties of Malva sylvestris leaves in constipation treatment. Biomed Pharmacother 2017;89:29-35.

15. Worley B, Powers R. PCA as a practical indicator of OPLSDA model reliability. Curr Metabolomics 2016;4:97-103.

16. Wan JB, Bai X, Cai XJ, et al. Chemical differentiation of Da-Cheng-Qi-Tang, a Chinese medicine formula, prepared by traditional and modern decoction methods using UPLC/Q-TOFMS-based metabolomics approach. J Pharm Biomed Anal 2013;83:34-42.

17. Ma N, Karam I, Liu XW, et al. UPLC-Q-TOF/MS-based urine and plasma metabonomics study on the ameliorative effects of aspirin eugenol ester in hyperlipidemia rats. Toxicol Appl Pharmacol 2017;332:40-51.

18. Gong YX, Wang H, Hou Y, et al. Effects of High-dose Raw Rhizoma Atractylodis Macrocephala Combined with Fructus Aurantii Immaturus on the Expression of 5-HT3R and 5-HT4R in Colon of Rats with Slow Transit Constipation.
Chin J Integr Tradit West Med (Chin) 2019;39:988-92.

19. Deloose E, Verbeure W, Depoortere I, et al. Motilin: from gastric motility stimulation to hunger signalling. Nat Rev Endocrinol 2019;15:238-50.

20. Meng XM, Liu XT. Effect of application of herbal medicine paste to "Tianshu" (ST25) on intestinal mobility and expression of vasoactive intestinal peptide and substance $\mathrm{P}$ in colonic myenteric plexus in rats with functional constipation. Zhen Ci Yan Jiu 2019; 44:906-10.

21. El Moussawi L, Chakkour M, Kreydiyyeh SI. Epinephrine modulates $\mathrm{Na}+\mathrm{K}+$ ATPase activity in Caco-2 cells via Src, p38MAPK, ERK and PGE2. PLoS One 2018;13:e0193139.

22. Graham HK, Maina I, Goldstein AM, et al. Intestinal smooth muscle is required for patterning the enteric nervous system. J Anat 2017;230:567-74.

23. Yu T, Wang Y, Qian D, et al. Advanced Glycation End Products Impair Ca2+ Mobilization and Sensitization in Colonic Smooth Muscle Cells via the CAMP/PKA Pathway. Cell Physiol Biochem 2017;43:1571-87.

24. Bessler H, Salman H, Bergman M, et al. Caffeine alters cytokine secretion by PBMC induced by colon cancer cells. Cancer Invest 2012;30:87-91.

25. Sturtzel B, Dietrich A, Wagner KH, et al. The status of vitamins B6, B12, folate, and of homocysteine in geriatric home residents receiving laxatives or dietary fiber. J Nutr Health Aging 2010;14:219-23.

26. Zhao XY, Wang JW, Yin Y, et al. Effect of Tong Xie Yao Fang on endogenous metabolites in urine of irritable bowel syndrome model rats. World J Gastroenterol 2019;25:5134-51.

27. Chen YF, Yu XJ, Liu XH, et al. Discussion on Biological Basis of Constipated Mice Cured by Optimal Prescription of Da Chengqitang with Differential Proteomics. Chin J Exp Med Formul 2017;23:93-9.

28. Gu X, Song Y, Chai Y, et al. GC-MS metabolomics on PPAR $\alpha$-dependent exacerbation of colitis. Mol Biosyst 2015;11:1329-37.

29. Singh K, Gobert AP, Coburn LA, et al. Dietary Arginine Regulates Severity of Experimental Colitis and Affects the Colonic Microbiome. Front Cell Infect Microbiol 2019;9:66.

Cite this article as: Yan S, Hao M, Yang H, Sun M, Wu B, Yue Y, Wang X. Metabolomics study on the therapeutic effect of the Chinese herb pair Fructus Aurantii Immaturus and Rhizoma Atractylodis Macrocephalae in constipated rats based on UPLC-Q/TOF-MS analysis. Ann Palliat Med 2020;9(5):2837-2852. doi: 10.21037/apm-20-280 